\title{
Exotische Atome: Rosen aus dem Blumengarten der subatomaren Physik
}

\author{
Paul Kienle ${ }^{a, b}$ und Johann Marton ${ }^{a}$ \\ ${ }^{a}$ Stefan Meyer Institut für subatomare Physik der ÖAW \\ $b$ Technische Universität München
}

\section{Einleitung}

Diese öffentlichen Vorlesung, im Rahmen der internationalen Konferenz Exotic Atoms 05, gibt einen elementaren Überblick über ein hochaktuelles Forschungsgebiet der subatomaren Physik, das der Physik stark wechselwirkenden Teilchen, der Hadronen. Nach einer kurzen Beschreibung von Wasserstoffatomen, werden elementare exotische wasserstoffähnliche Atome, einschließlich des Antiwasserstoffs vorgestellt. Ersetzt man das Elektron in einem Atom durch ein negativ geladenes Pion oder Kaon so entsteht ein pionisches oder kaonisches Atom. Pionen und Kaonen sind Quark-Antiquark Verbindungen und zeigen in exotischen Atomen neben der anziehenden elektromagnetischen Wechselwirkung auch eine starke Wechselwirkung kurzer Reichweite mit dem Kern des Atoms, der aus Protonen und Neutronen besteht, die anziehend oder abstoßend sein kann. Ferner werden Pionen und Kaonen im Kern absorbiert, wodurch die Energiezustände des exotischen Atoms kürzere Zeit leben und dadurch eine messbare Breite bekommen. Die Eigenzustände der pionischen und kaonischen Atome werden also auf Grund der starken Wechselwirkung gegenüber denen der rein elektromagnetischen verschoben und verbreitert. Durch sehr genaue Messung der Energie der Strahlung beim Übergang zwischen zwei Eigenzuständen, kann deren Verschiebung und Verbreiterung gemessen werden. Daraus leitet man dann die Parameter zur Beschreibung der Stärke der starken Wechselwirkung bei niedrigen Energien ab.

Es werden laufende Experimente beschrieben zur Messung der K-Röntgen Übergange im pionischen Wasserstoff, die zur Präzisionsbestimmung der Niederenergie Pion-Nukleon Wechselwirkung führen. Diese dient zum Vergleich mit Pion-Kern Wechselwirkungsstärken, die durch Messung der Bindungsenergien und Breiten von pionischen Grundzuständen schwerer Kerne gewonnen 
werden. Aus dem Verhältnis der Pion-Kern zur Pion-Nukleon Wechselwirkung können interessante Erkenntnisse zur besonderen Dynamik der PionWechselwirkung abgeleitet werden, die in direktem Zusammenhang stehen mit der spontanen chiralen Symmetrie-Brechung der starken Wechselwirkung, die zu den anomal niedrigen Massen des Pions und den hohen des Nukleons führt.

Analog zur Pion Wechselwirkung wurde die des Antikaons mit einem Nukleon durch Spektroskopie der K Röntgen-Übergänge im kaonischen Wasserstoff kürzlich untersucht. Sie ist auf Grund einer Resonanz unterhalb der AntikaonProton Schwelle abstoßend und unterhalb der Resonanz stark anziehend, was zu einem kürzlich entdeckten sehr interessanten Phänomen führt. Lagert man negative Antikaonen in leichte Kerne ein, so führen sie zu stark gebundenen Kernzuständen mit hoher Dichte, bei der man einen interessanten Phasenübergang zu einem supraleitenden Quark-Gluon Plasma oder Kaon-Kondensation erwartet. In ersten Experimenten wurde ein kaonisches Tribaryon gefunden mit der hohen Bindungsenergie von etwa $200 \mathrm{MeV}$.

\section{Was sind exotische Atome?}

Zur Beantwortung dieser Frage betrachten wir zuerst ein normales Wasserstoffatom. Es besteht aus einem positiv geladenen Proton in dessen elektrischen Feld ein negativ geladenes Elektron mit einer Bindungsenergie $E=\alpha^{2} m_{e} c^{2} / 2 \sim 13.6 \mathrm{eV}$ gebunden ist. Dabei ist $\alpha=1 / 137$, die Kopplungskonstante der elektromagnetischen Wechselwirkung und $m_{e} c^{2}$ $=511 \mathrm{keV}$, die der Ruhemasse entsprechende Energie des Elektrons. Ein Maß für die Ausdehnung der Elektronen-Wolke um das Proton ist der Bohr-Radius der $\sim 1 / m_{e} c^{2} \sim$ $50.000 \mathrm{fm}\left(1 \mathrm{fm}=10^{-15} \mathrm{~m}\right)$ ist.

Ersetzt man das Elektron durch ein negatives exotisches Teilchen, so entsteht ein exotisches Wasserstoffatom. Ist das Teilchen ein schweres Elektron, also ein Myon, das ungefähr 200-mal schwerer als das Elektron ist, so ist die Bindungsenergie von myonischem Wasserstoff entsprechend höher, also ungefähr $2.72 \mathrm{keV}$ und die Ausdehnung der Myon Wolke nur $250 \mathrm{fm}$. In myonischen Atomen schwerer Elemente überlappt die Myon Wolke mit der Verteilung der Protonen in ausgedehnten Kernen. Durch genaue Messung der Myon Bindungsenergie konnten die Radien der Ladungsverteilung von Kernen $r_{B} \sim 1 /\left(Z m_{\mu} c^{2}\right)$ vermessen werden. Myonen können auch Moleküle wie z.B. das DT-Molekül stark binden. Dies führt zu einem Überlapp der Deuterium und Tritium Kerne und damit zur Myon katalysierten kalten Fusion zu ${ }^{4} \mathrm{He}$ mit hohem Energie-Gewinn, da ein Myon eine lange Kette von Reaktionen katalysieren kann bis es schließlich an einem ${ }^{4} \mathrm{He}-\mathrm{Kern}$ hängen bleibt und für die Katalyse verloren geht. Bisher ist die Energieerzeugung in der Myonkatalysierten Fusion niedriger als aufgewandt werden muss, um ein Myon zu erzeugen, was eine technische Anwendung noch verhindert.

Das exotische Atom, das bei seiner ersten Synthese vor knapp 10 Jahren die meisten Schlagzeilen machte, ist der Antiwasserstoff, der aus einem negativ geladenen Antiproton und ein daran gebundenem positiv geladenen Positron besteht, also den Antiteilchen des Protons und des Elektrons. Der Antiwasserstoff sollte bei der Gültigkeit der Teilchen- 

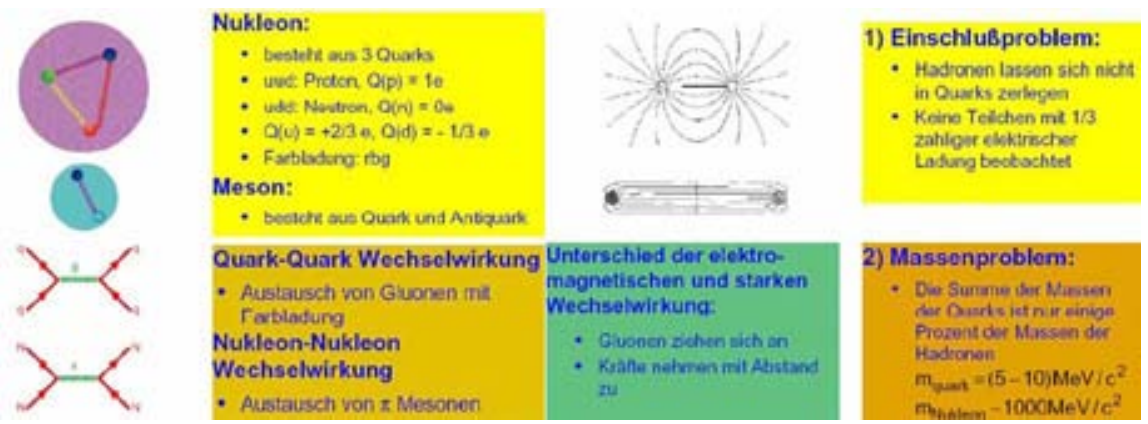

Fig. 1: Aufbau der Nukleonen und Mesonen aus Quarks und Gluonen und die Besonderheiten von Hadronen.

Antiteilchen Symmetrie die gleichen Eigenschaften haben wie der Wasserstoff, also gleiche Massen, gleiche Bindungsenergien usw. Primäre Antimaterie findet man aber nicht in unserer Galaxie. Beim Urknall sollte sie aber mit gleicher Wahrscheinlichkeit wie die Materie aus der Strahlung entstanden sein. Wo ist sie geblieben? In anderen Galaxien oder gibt es eine Brechung der Symmetrie? Nach der wird am CERN durch Vergleich der Spektren von Antiwasserstoff und Wasserstoff gesucht.

\section{Der Aufbau von Hadronen - pionische und kaonische Atome}

Ersetzt man in einem Atom das Elektron durch ein negativ geladenes Pion oder Kaon, so erhält man ein pionisches oder kaonisches Atom. Pionen und Kaonen sind Mesonen, durch die starke Wechselwirkung gebundene Quark-Antiquark Paare, die neben der anziehenden elektromagnetischen Wechselwirkung (bei negativer Ladung) auch eine starke Wechselwirkung mit Nukleonen und Kernen zeigen, die anziehend oder abstoßend sein kann.

Die Bausteine der Hadronen, der stark wechselwirkenden Teilchen, sind die Quarks und Antiquarks, die durch den Austausch von Gluonen verbunden sind. Die Nukleonen sind aus drei Valenzquarks aufgebaut, von denen es zwei Arten gibt, die drittelzahlige elektrische Ladungen tragen, die up(u) und down(d) Quarks mit $+2 / 3 e(u)$ und $-1 / 3 e(d)$ elektrischen Elementarladungen. Dazu trägt jeder Quark eine starke Ladung (Farbladung), von denen es drei Sorten gibt, die mit drei Farben gekennzeichnet sind: rot, grün, blau. $\mathrm{Zu}$ jedem Quark gibt es ein Antiquark, mit umgekehrter elektrischer und komplementärer Farbladung. Die aus Quarks zusammengesetzten Hadronen sind farbneutral, zeigen nach außen keine starke Ladung Nach diesem Bauprinzip, enthalten Nukleonen 3 Quarks mit den Farbladungen rot, grün und blau, was nach außen das farbneutrale weiß ergibt. Mesonen sind, wie gesagt aus Quark-Antiquark Paaren aufgebaut, die eine Farb- und eine komplementäre Antifarb-Ladung tragen. Die Wechselwirkung zwischen den Quarks (An- 

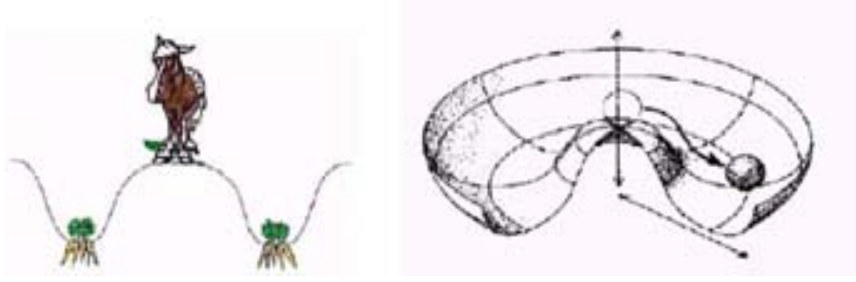

Fig. 2: Spontane Symmetriebrechung.

tiquarks), wird von massenlosen Feldquanten, den Gluonen, übertragen, analog zu den $\gamma$-Quanten der elektromagnetischen Wechselwirkung. Es besteht jedoch ein wesentlicher Unterschied, während die $\gamma$-Quanten ladungsneutral sind, tragen die Gluonen eine Farbladung und eine Antifarbladung, sind also nicht ladungsneutral und ziehen sich daher an. Dies führt letztlich zum Einschluss der Quarks. Es wurden bisher keine einzelne Quarks, sondern nur aus Quarks aufgebaute farbneutrale Hadronen gefunden, von denen es zwei Arten gibt mit denen wir uns im weiteren beschäftigen wollen, den Protonen (uud) und Neutronen (udd) sowie den negativen Pionen $(\bar{u} \mathrm{~d})$ und negativen Kaonen $(\bar{u} \mathrm{~s})$, wobei die Kaonen einen seltsamen (s)-Quark mit -1/3e elektrischer Ladung enthalten. Kerne sind aus Nukleonen aufgebaut, die im Wesentlichen durch den Austausch von Pionen gebunden sind.

Neben dem Einschlussproblem der Quarks in Hadronen tritt noch eine andere Anomalie auf. Während normale gebundene Systeme, wie z.B. Kerne, immer niedrigere Massen haben wie die Summe der Massen ihrer Bausteine, z.B. der Nukleonen, ist es bei Hadronen genau umgekehrt, Das Nukleon hat eine rund 30mal größere Masse als die Summe der Massen der 3 Quarks aus denen es aufgebaut ist. Hier ist ein völlig anderes Prinzip der Massen Generierungen am Werk als wir es von normalen gebundenen Systemen gewohnt sind.

\section{Spontane chirale Symmetriebrechung}

Johannes Buridian (1300-1358), Scholastiker und Rektor der Universität Paris stellte folgende im Fig. 2 links skizzierte Frage: Verhungert der Esel auf dem kahlen Bergrücken obwohl in den völlig symmetrisch rechts und links gelegenen Tälern Nahrung für ihn wächst? Nein, er bricht die rechts-linke Symmetrie spontan und geht entweder in das linke oder rechte Tal hinunter.

Die rechte Seite von Fig. 2 zeigt eine physikalische Version des Buridianschen Problems. Eine Kugel liegt auf dem Symmetrie Punkt eines mexikanischen Hutes, der auch ein Potential einer Wechselwirkung darstellen soll. Die Kugel bricht spontan die Symmetrie und rollt in irgendeiner Richtung zu einem energetisch niedrigeren Zustand, dem Grundzustand des Potentials. Die Kugel kann aber jeden Ort in der Potentialmulde einnehmen, 
was bedeutet, dass es einen mit dem Grundzustand energetisch entarteten zusätzlichen Zustand gibt. Kippt man den Hut, so rollt die Kugel wieder in eine Richtung, kann aber um den tiefsten Zustand schwingen, die Entartung des Grundzustands ist aufgehoben. Spontane Symmetrie Brechung spielt eine wichtige Rolle in der Dynamik wechselwirkenden Systeme wie den magnetischen Momenten magnetischer Materialien. Heisenberg wies darauf hin, dass in magnetischen Stoffen, wie Eisen, unterhalb des Curiepunkts die isotrope Richtungsverteilung der magnetischen Momente spontan gebrochen wird und ein energetisch niedriger Zustand durch Ausrichtung der Momente sich einstellt.

Nambu und Jona-Lasino haben die spontane Brechung der chiralen Symmetrie masseloser Quarks, auf Grund ihrer starken Wechselwirkung, als Ursache für die Erzeugung der hohen Massen von Hadronen und der niedrigen der Pionen vorgeschlagen. Ein anschauliches Bild einer chiralen Symmetrie, also dem Drehsinn, findet man im Alltag einer gedeckten Tafel, bei der die Salate exakt symmetrisch zwischen den Haupttellern gestellt wurden, also symmetrisch zwischen rechts und links. Niemand in der Tafelrunde wagt zum Salat zu greifen, bis einer sich spontan entscheidet die Symmetrie zu brechen und das linke Salatteller zu sich herzuholen, alle anderen werden ihm folgen. Im Übrigen gibt es für diese chirale Symmetriebrechung eine nützliche Regel: Salat links, Dame rechts!

Masselose Teilchen mit Spin 1/2 sind unabhängig von ihrem Drehsinn, links oder rechts in Bezug auf ihre Bewegungsrichtung, in ihrer Wechselwirkung gleich. Man sagt sie besitzen chirale Symmetrie. Quarks besitzen aber eine kleine Masse, die die chirale Symmetrie direkt bricht. Man kann immer ein Bezugsystem finden, das sich mit Lichtgeschwindigkeit bewegt in dem das Teilchen mit Masse, das sich langsamer bewegt, den Drehsinn seines Spins umdreht, also die chirale Symmetrie bricht. Darüber hinaus brechen Quarks durch ihre starke Wechselwirkung die chirale Symmetrie spontan. Es entsteht, wie im Fig. 3 gezeigt wird, ein energetisch tiefer liegender Grundzustand, der Vakuumzustand.

Die Hadronen, Mesonen und Nukleonen sind angeregte Zustände, die durch eine Energieoder Massenlücke vom Grundzustand getrennt sind, wobei die Energie im Gluonenfeld steckt. Die Pionen (und auch Kaonen) sind die Ausnahme. Bei masselosen Quarks wären sie energetisch entartet mit dem Grundzustand, also dem Vakuum. Nur durch die kleine Masse der Quarks, die verstärkt wird durch die spontane chirale Symmetriebrechung, erhalten die Pionen ihre Masse von $140 \mathrm{MeV} / \mathrm{c}^{2}$, die klein ist gegenüber einer typischen Nukleonmasse von $\sim 1000 \mathrm{MeV} / \mathrm{c}^{2}$. Man nennt diese fast masselosen Vakuumanregungen nach ihren Erfindern Nambu-Goldstone Bosonen.

Das Vakuum, also der Grundzustand, ist gefüllt mit Antiquark-Quark Paare hoher Dichte $\left(1.5 \mathrm{fm}^{-3}\right.$, was rund 10 mal höher als die Kerndichte ist) und als chirales QuarkKondensat bezeichnet wird. Als Ordnungsparameter für diesen Phasenübergang wird der Vakuumerwartungswert des Quarkkondensats $<\bar{q} q>$ oder äquivalent $f_{\pi}^{2}$ benutzt, das Quadrat der Pion-Zerfallskonstante $f_{\pi}$, das gemäß der Gellman, Oaks, Renner Beziehung proportional dem Erwartungswert des Quarkkondensats ist (siehe Fig. 3). Füllt man in das Vakuum einen Kern, so wird das Quarkkondensat und entsprechend $f_{\pi}^{2}$ reduziert (siehe rechtes Diagramm von Fig. 3). Unsere Forschung befasst sich mit der chiralen Dynamik, der starken Wechselwirkung der Nambu-Goldstone Bosonen, also den Pionen und Kaonen, mit den Nukleonen bei niedrigen Energien. 


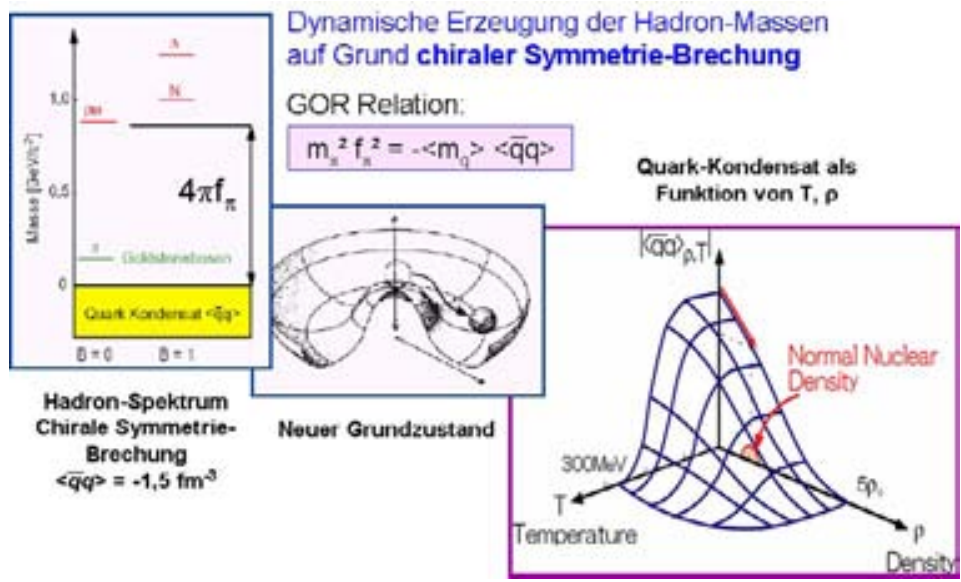

Fig. 3: Brechung der chiralen Symmetrie von Quarks durch die starke Wechselwirkung.

Bei den Pionen ist die Wechselwirkung an der Schwelle relativ schwach und wird durch den Ordnungsparameter der spontanen Symmetrie Brechung $f_{\pi}^{2}$ bestimmt. Dazu untersucht man am einfachsten die Energieverschiebung und Breite des tiefsten Zustands (1s) eines pionischen Atoms mithilfe von Röntgenspektroskopie. Das elementarste pionische Atom zum Studium der Pion-Nukleon Wechselwirkung ist pionischer Wasserstoff. Bei Atomen mit einer Ordnungszahl höher als $\mathrm{Z}=15$ werden die 1s-Zustände nicht mehr bevölkert, das Pion wird vom Kern schon in höheren Zuständen absorbiert. Wir entwickelten zum Studium solcher tiefgebundenen 1s-Zuständen eine neue Methode, die der rückstossfreien Pion Transfer-Reaktionen direkt in die tiefliegenden 1s- oder 2p-Zustnde zur Bestimmung der stark geänderten Bindungsenergie und der relativ großen Breiten der 1s-Zustände in schweren Kernen, wie Zinn und Blei. Durch Vergleich der Pion-Nukleon und Pion-Kern Wechselwirkung wurde eine teilweise Restaurierung der spontan gebrochenen chiralen Symmetrie durch ein Kernmedium im Vakuum beobachtet.

Bei kaonischem Wasserstoff wird die Kaon-Nukleon Wechselwirkung im Gegensatz zur Pion-Nukleon Wechselwirkung durch eine Resonanz $(\Lambda(1405)), 27 \mathrm{MeV}$ unterhalb der Kaon-Proton Schwelle, bestimmt. Damit wird die chirale Dynamik überdeckt und man erwartet oberhalb der Resonanz, also im Bereich des 1s-Zustands von kaonischem Wasserstoff eine abstoßende Wechselwirkung, unterhalb der Resonanz tiefgebundene Zustände. Dies führt zu einer hochinteressanten Möglichkeit mithilfe der starken anziehenden Wechselwirkung von negativen Kaonen, hoch verdichtete Kerne zu erzeugen. Damit könnte es gelingen das Phasen Diagramm von Kernmaterie im Bereich der Dichte von Neutronen Sterne sowie einem Phasenübergang zu einer besonderen Form des Quark-Gluon Plasmas $\mathrm{zu}$ untersuchen. 


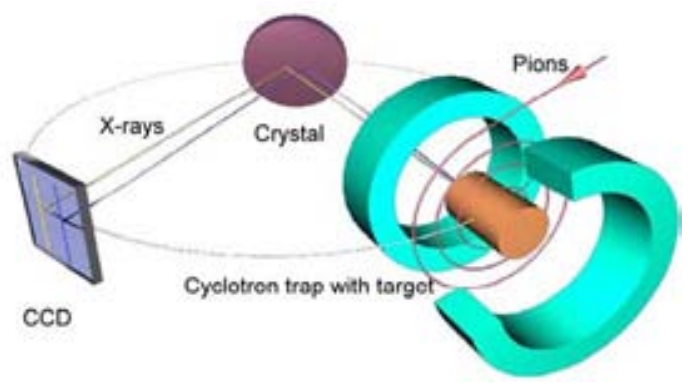

Fig. 4: Anordung zur Präzisionsmessung der K-Röntgenstrahlung von pionischem Wasserstoff.

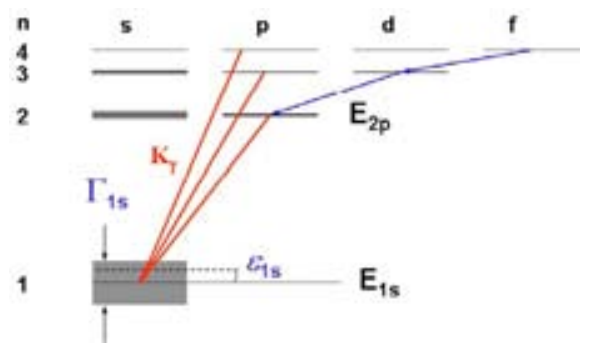

Fig. 5: Röntgenübergänge in einem pionischen Wasserstoffatom.

\section{Pionischer Wasserstoff}

Negative Pionen werden durch Beschuss eines Kohlenstoff-Targets mit einem Protonenstrahl erzeugt, der im Sektorzyklotron des Paul Scherrer Instituts (PSI) auf $600 \mathrm{MeV}$ beschleunigt wird. Mit Hilfe einer kurzen Strahlführung werden die kurzlebigen Pionen $\left(10^{-8} \mathrm{~s}\right)$ auf eine spezielle Wasserstoff-Target Anordnung gelenkt (Fig. 4).

Sie werden in einem Magnetfeld in Folien abgebremst und dadurch auf Bahnen wie in einem umgekehrten Zyklotron auf ein radial konzentriertes Wasserstoff-Target gelenkt, dort in eine gebundene Bahn eingefangen und kaskadieren dann unter Emission von Strahlung zu den $\mathrm{n}=2$, 3, 4 p-Zuständen, die durch Aussendung von K-Röntgenstrahlung zum 1sGrundzustand zerfallen. Deren Energie ist im Wesentlichen durch die Differenz der elektromagnetischen Energien bestimmt und einer kleinen Verschiebung und Verbreiterung des 1s-Zustandes durch die starke Wechselwirkung (Fig. 5).

Die Energien der K Röntgen-Übergänge werden mit einem Kristall-Spektrometer gemessen. Dabei werden die Strahlen an den Gitterebenen eines Kristalls unter Winkeln reflektiert, die charakteristisch sind für die Wellenlänge der Strahlung und mit einem ortsempfindlichen Detektor nachgewiesen. Mit dieser Methode können die relevanten Rönt- 


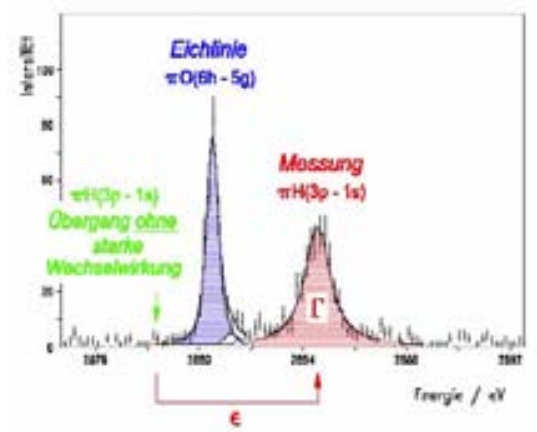

Fig. 6: Röntgenlinie von pionischem Wasserstoff $\left(\mathrm{K}_{\beta}, 3 \mathrm{p}-1 \mathrm{~s}\right)$ und Sauerstoff-Eichlinie.

$$
\begin{gathered}
\begin{array}{c}
\text { Chirales Niederenergie-Theorem } \\
\text { (Tomozawa-Welnberg) }
\end{array} \\
T^{(+)}=\frac{1}{2}\left(T_{z_{p} p}+T_{z^{n} n}\right)=4 \pi \varepsilon_{1} b_{0}=0 \quad \text { isoskalar } \\
T^{(-)}=\frac{1}{2}\left(T_{n, p}-T_{n n}\right) \equiv-4 \pi \varepsilon_{1} b_{1}=\frac{\omega}{2 f_{n}^{2}} \quad \text { isovektor }
\end{gathered}
$$

Fig. 7: Pion-Nukleon Streulängen.

genstrahlenergien mit höchster Präzision bestimmt werden (siehe Fig. 6).

Das Röntgen Spektrum zeigt die verbreiterte 3p-1s Linie, die etwa 7eV höhere Energie besitzt, als der erwartete Übergang ohne starke Wechselwirkung, die also erwartungsgemäß anziehend ist. Ziel dieses Experiments, das vor dem Abschluss steht, ist die verbesserte Messung der isoskalaren und iso-vektor Streulängen der Pion-Nukleon Wechselwirkung und ihr Vergleich mit theoretischen Voraussagen. Besonders interessant ist die Neubestimmung der kleinen isoskalaren Streulänge, die besagt, dass die Pion-Proton Streulänge gleich stark ist wie die Pion-Neutron Wechselwirkung, aber umgekehrtes Vorzeichen besitzt. Die isovektor Streulänge hingegen ist im Wesentlichen durch $f_{\pi}^{2}$, dem Ordnungsparameter der chiralen Symmetrie Brechung bestimmt (siehe Fig. 7).

\section{Pion im Kern}

Wasserstoffähnliche Zustände von einem Pion mit einem Kern als Zentrum dienten seit langer Zeit zur Untersuchung der Pion-Kern Wechselwirkung. Dazu wurde analog zum pionischen Wasserstoff die Röntgen-Spektroskopie des pionischen Übergangs benutzt. Auf Grund der mit der Ordnungszahl zunehmenden Absorption der Pionen konnten 1s 


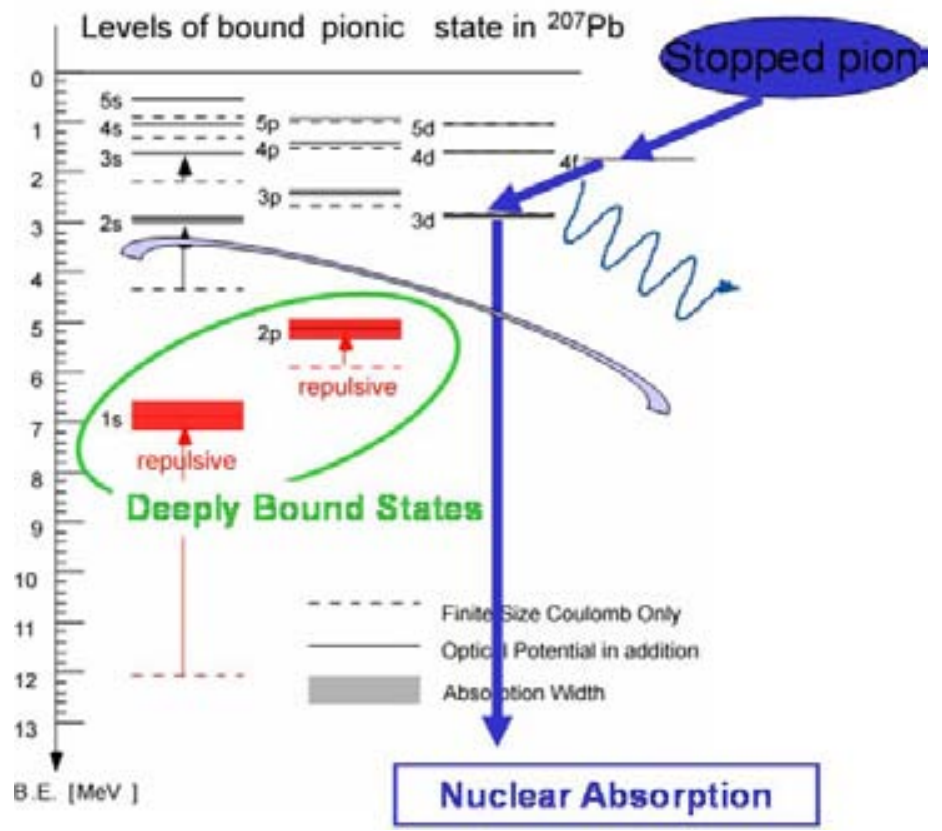

Fig. 8: Energiezustände von pionischem ${ }^{207} \mathrm{~Pb}$.

Zustände nur bis $Z=15$ beobachtet werden. Ein Vergleich der Pion-Kern Wechselwirkung mit der Pion-Nukleon Wechselwirkung ergibt eine interessante Möglichkeit der Frage nachzugehen, ob die Pion-Kern Wechselwirkung stärker ist als die Pion-Nukleon Wechselwirkung, insbesondere sein isovektor Anteil der umgekehrt proportional zu $f_{\pi}^{2}$ ist, dem Ordnungsparameter der spontanen Symmetriebrechung. Dieser Anteil kann am besten in schweren Kernen mit einem Neutronen-Überschuss untersucht werden, da er proportional der Differenz der Neutronen- und Protonen-Dichte im Kern ist. Der Nachteil besteht aber darin, dass in schweren Kernen die Röntgenkaskaden bei Zuständen mit hohen Drehimpulsquantenzahlen enden und der 1s Zustand wegen der Absorption nicht erreicht wird (siehe Fig. 8).

In einem ${ }^{207} \mathrm{~Pb}$ Kern ist der letzte beobachtbare Röntgenübergang zwischen den $4 \mathrm{f}$ und 3d Zuständen, mit sehr kleinem s-Wellen Anteil. Daher musste ein neues Werkzeug erfunden werden, die tiefgebundenen 1s- und 2p-Zustände zu bevölkern und zu spektroskopieren (siehe Fig. 9).

Wir benutzten die $\left(\mathrm{d},{ }^{3} \mathrm{He} \pi^{-}\right)$Transfer Reaktion. Bei einer Einschuss-Energie von etwa $500 \mathrm{MeV}$ wird das Pion ohne Impulsübertrag auf den Target-Kern übertragen, gleichzeitig ein Neutron des Target Kerns in ein Proton verwandelt und an das Deuteron angelagert, das dann als ${ }^{3} \mathrm{He}$ nach vorwärts ausgesandt wird. Bei genau bekannter Einschussenergie 

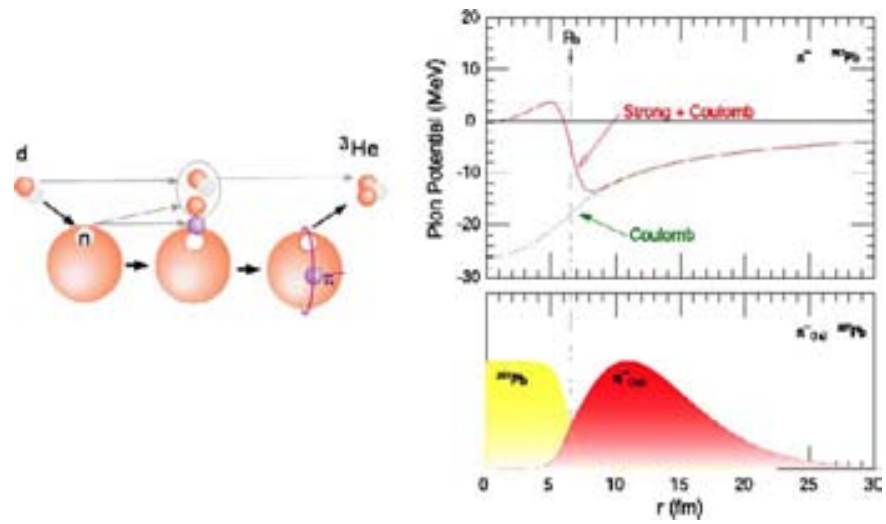

Fig. 9: Schematische Darstellung einer $\left(\mathrm{d},{ }^{3} \mathrm{He} \pi^{-}\right)$pionischer Transferreaktion (links) und des Potentials und der Pionverteilung in einem pionischen ${ }^{207} \mathrm{~Pb}$ Atom.

und entsprechend genauer Messung der ${ }^{3}$ He-Energie, kann die Anregungsenergie des gebildeten pionischen Kerns und damit seine Bindungsenergie und Breite bestimmt werden. Wie theoretisch erwartet ist die starke Wechselwirkung in Kernen mit Neutronen-Überschuss abstoßend. Aus der Überlagerung mit der anziehenden Coulomb-Wechselwirkung entsteht am Kernrand eine Potentialtasche in der das Pion gebunden ist und einen maximalen Überlapp mit der Kerndichteverteilung bei ungefähr 0.6-mal der Dichte vom Kerninnern hat. Dadurch wird die Linien-Breite auf Grund der Pion Absorption wesentlich reduziert, was zu energetisch gut getrennten 1 s und $2 \mathrm{p}$ pionischen Zuständen führt. Die verwendete experimentelle Technik zur Beobachtung tiefgebundener pionischer Zustände ist in Fig. 10 skizziert. Ein intensiver, monoenergetischer $500 \mathrm{MeV}$ Deuteronen Strahl trifft auf ein dünnes Target $\left(20 \mathrm{mg} / \mathrm{cm}^{2}\right)$. Die ohne großen Impulsübertrag nach vorwärts ausgesandten ${ }^{3}$ He Kerne werden im ersten Teil eines magnetischen Doppel-Spektrometers nach Impulsen örtlich in einer Fokalebene getrennt und mit ortsempfindlichen Driftkammern spektroskopiert. Im zweiten Teil des Spektrometers werden die ${ }^{3} \mathrm{He}$ Kerne nach einem Energieverlust in einem Szintillator abgetrennt und mithilfe von Szintillatoren am Ende des Spektrometers untergrundfrei nachgewiesen. Fig. 11 zeigt die Energiespektren der ${ }^{3} \mathrm{He}$ Kerne von ${ }^{124,120,116}$ Sn Target-Kernen zusammen mit Eichspektren aus einer $\mathrm{d}+\mathrm{p} \rightarrow{ }^{3} \mathrm{He}$ $+\pi^{0}$ Reaktion, die monoenergetische ${ }^{3} \mathrm{He}$ Linien genau bekannter Energien erzeugt. Die Spektren zeigen überdies Linien, die pionischen 1s Zuständen in den ${ }^{123,119,115}$ Sn Isotopen zugeschrieben werden können. Aus den Bindungsenergien und Breiten der pionischen 1s Zustände der Sn Isotope und entsprechenden Daten von 1s Zuständen leichter isoskalaren $(\mathrm{Z}=\mathrm{N})$ Kerne wurden alle relevanten Pion-Kern s-Wellen Wechselwirkungsparameter bestimmt und die isovektor Pion Streulänge mit der vom pionischen Wasserstoff abgeleiteten verglichen (Fig. 11 rechts). Dieser Vergleich ergab, dass ein Kernmedium mit der Dichte $\rho(0)=0.17 \mathrm{fm}^{-3}$ die Dichte des chiralen Quarkkondensats um $33 \%$ reduziert. In der chiralen Dynamik wird der isovektor Teil der Pion-Kern Wechselwirkung gegenüber dem 


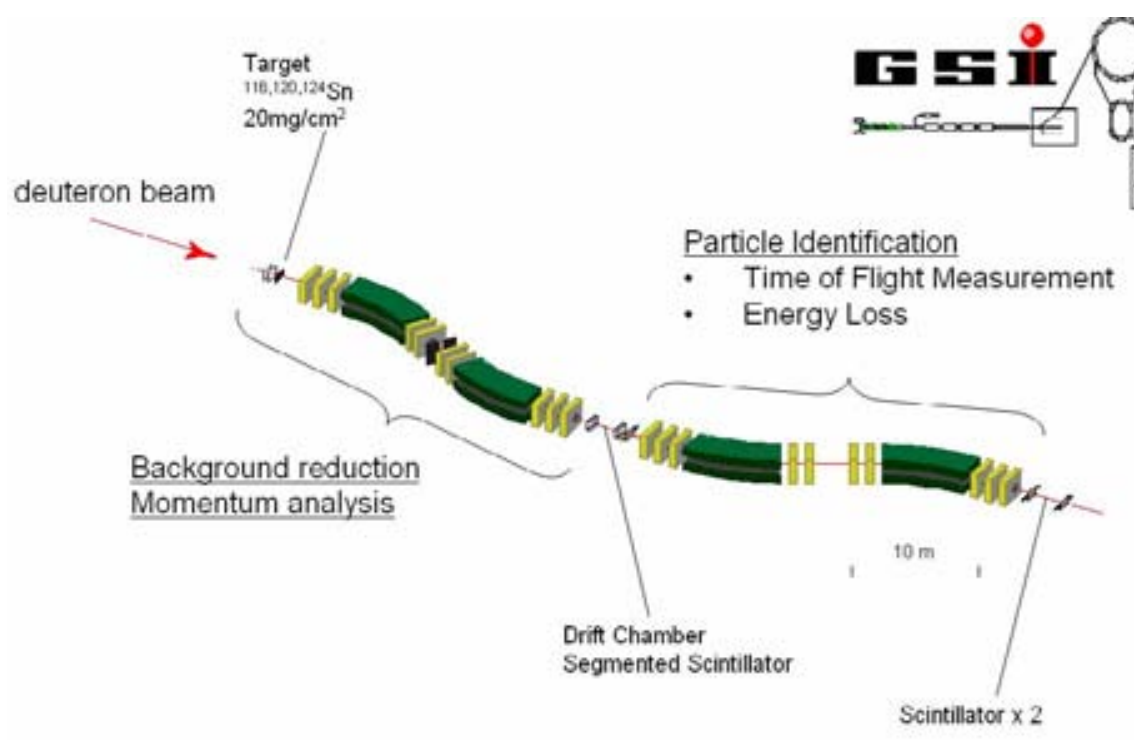

Fig. 10: Experimentelle Technik zu Beobachtung tiefgebundener Zustände.

Pion-Nukleon Anteil, entsprechend der Reduzierung von $f_{\pi}^{2}$ in einem nuklearen Medium, erhöht. Dies zeugt von der theoretisch erwarteten partiellen Restaurierung der spontan gebrochenen chiralen Symmetrie von wechselwirkenden Quarks durch ein nukleares Medium.

\section{Kaonischer Wasserstoff \\ - s Quark in der starken Wechselwirkung}

Kaonen und deren Antikaonen können ähnlich wie die Pionen als Nambu-Goldstone Bosonen der starken Wechselwirkung mit s Quarks gesehen werden. Auf Grund der hohen direkten Masse der s Quarks (80-130 MeV/c $)$ ist die durch chirale Symmetriebrechung verstärkte Masse der Kaonen schon relativ hoch $\left(500 \mathrm{MeV} / \mathrm{c}^{2}\right)$. Trotzdem können sie, weniger genau, im Rahmen einer chiralen Störungstheorie behandelt werden. Das negative Kaon $(\bar{u}$ s), mit dem kaonischer Wasserstoff gebildet wird, ist ein Antiteilchen zum positiven Kaon $(u \bar{s})$, ein grundsätzlicher Unterschied zu den Pionen, die nur aus leichten Quarks bestehen. Dies zeigt sich auch in der Wechselwirkung der Antikaonen mit Nukleonen, die allein aus Quarks bestehen und somit auf Grund des Pauli Prinzips keine 

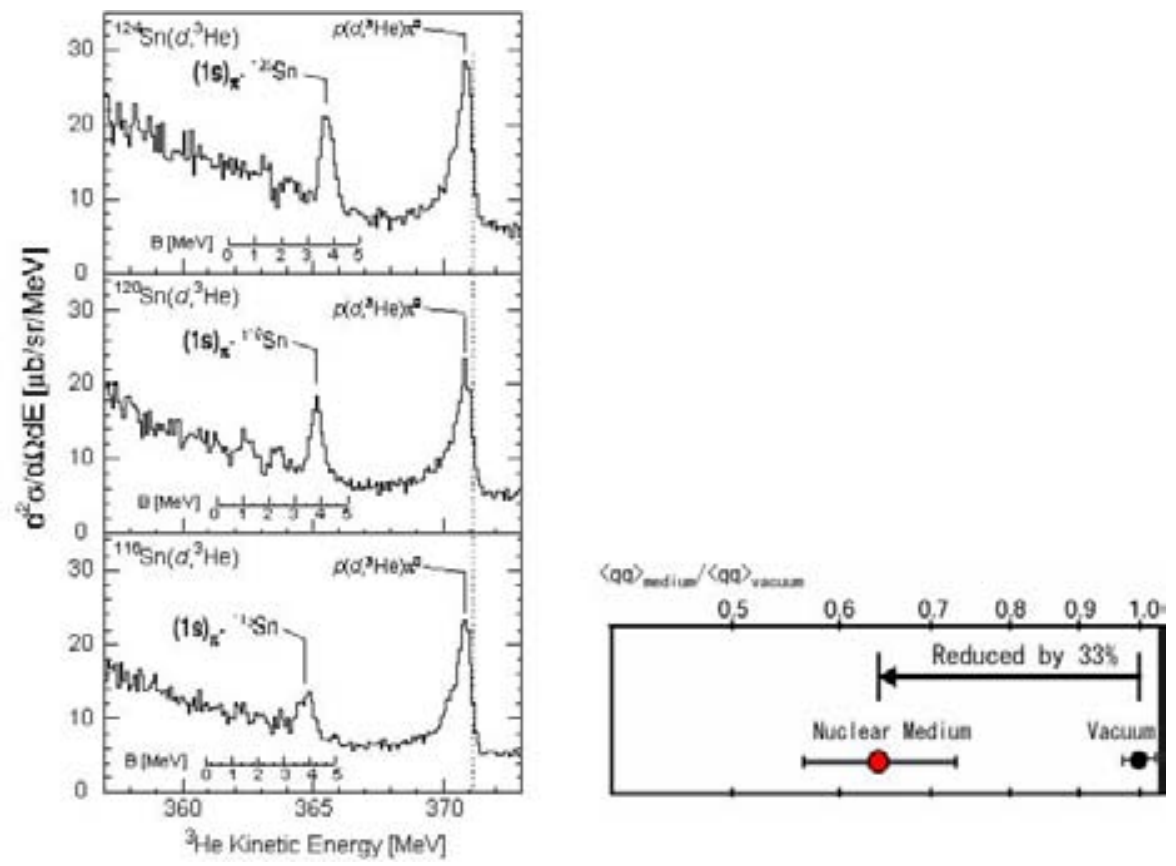

Fig. 11: ${ }^{3} \mathrm{He}-$ Spektren der $\left(\mathrm{d},{ }^{3} \mathrm{He} \pi^{-}\right)$Reaktion an Sn Targets (links). Vergleich der Isovektor-Streulängen (rechts).

abstoßende Wechselwirkung mit den $(\bar{u} \mathrm{~s})$-Quarks des $\mathrm{K}^{-}$Mesons erfahren.

Kürzlich wurde das DEAR Experiment zur Röntgen Spektroskopie von kaonischem Wasserstoff in Frascati abgeschlossen. Mit dem DAФNE $\left(\mathrm{e}^{+} \mathrm{e}^{-}\right)$-Collider wurden in Resonanz $\Phi$-Mesonen erzeugt, die mit hoher Wahrscheinlichkeit in ein Paar von $\mathrm{K}^{+} \mathrm{K}^{-}$Mesonen zerfallen. Die $\mathrm{K}^{-}$Mesonen wurden in einem gekühlten Wasserstoff-Target abgebremst und eingefangen. Die bei der Abregung ausgesandten Röntgen-Strahlen wurden mit einer aus 16 CCD Elementen bestehenden Anordnung, die das Target umgab, nachgewiesen und ihre Energie mit 160 eV Aufösung gemessen (Fig. 12).

Das kaonische Wasserstoff K-Röntgenspektrum ist nach Abzug des hohen Bremsstrahl Untergrunds in Fig. 13 dargestellt. Es zeigt eine abstoßende $\mathrm{K}^{-} \mathrm{p}$ Wechselwirkung, mit einer Verschiebung von $(-193 \pm 46) \mathrm{eV}$ und einer Linienbreite von $(249 \pm 142) \mathrm{eV}$. In Übereinstimmung mit dem Ergebnis der KpX Kollaboration wurde somit die abstoßende Wechselwirkung bestätigt, jedoch konnte eine höhere Genauigkeit der Verschiebung und Breite erzielt werden.

Diese abstoßende Antikaon-Nukleon Wechselwirkung nahe der Schwelle ist durch die $\Lambda(1405)$ Resonanz verursacht, wie quantitativ mit Hilfe einer gekoppelten Kanal-Theorie von Weise und Mitarbeiter schon vor 10 Jahren vorausgesagt wurde (siehe Fig. 14). 


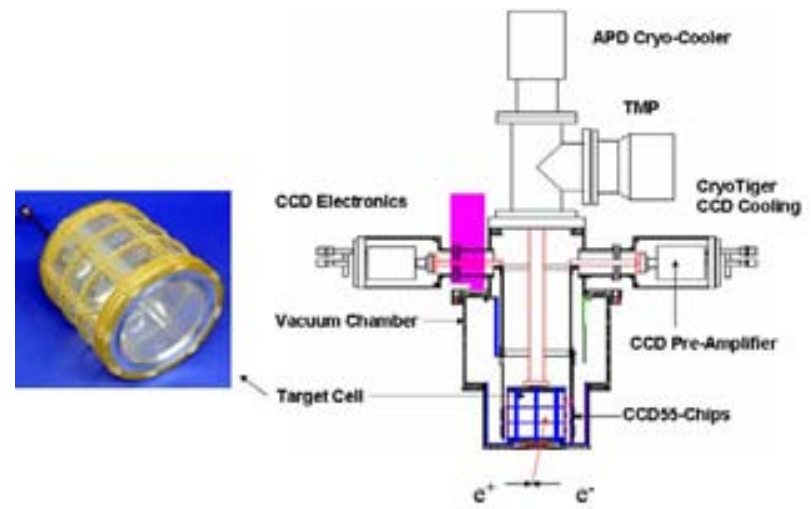

Fig. 12: Anordnung zur Röntgenspektroskopie von kaonischem Wasserstoff.

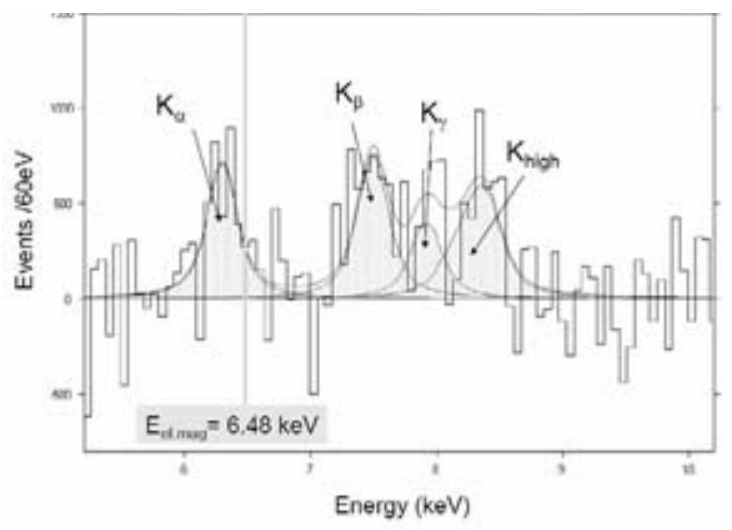

Fig. 13: Röntgenspektrum von kaonischem Wasserstoff. 


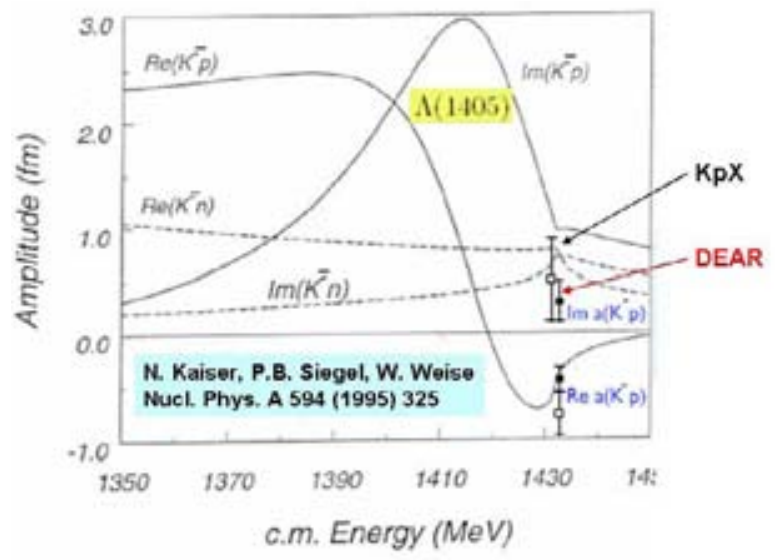

Fig. 14: Amplitude der $\mathrm{K}^{-}$-Proton und -Neutron Wechselwirkung in der Nähe der $\Lambda(1405)$ Resonanz.

Aktuell an dieser Voraussage ist die stark anziehende Antikaon-Proton Wechselwirkung unterhalb der Resonanz. Akaishi und Yamazaki sagten voraus, dass diese Wechselwirkung zu stark gebundenen Zuständen leichter Nukleonsysteme führt, die ohne Antikaonen teilweise nicht gebunden wären.

\section{Antikaonen im Kern}

Das elementarste mit $\mathrm{K}^{-}$gebundene System ist ein exotisches Wasserstoff Molekül aus zwei Protonen, in dem eine $\mathrm{K}^{-}$Meson die Austauschrolle des Elektrons übernimmt.

In Fig. 15 ist das ppK ${ }^{-}$Molekül dargestellt, so wie es von Akaishi und Yamazaki mit einem nichtrelativistischen Potentialmodell berechnet wurde, ausgehend von der $\Lambda(1405)$ Resonanz als gebundener Zustand von Proton und $\mathrm{K}^{-}$, mit empirisch bestimmten Parametern, die aus der Bindungsenergie der $\Lambda(1405)$ Resonanz, der $K^{-} p$ Streulänge und der Verschiebung und Breite des 1s Zustands von kaonischem Wasserstoff abgeleitet wurden. Mit diesem Potentialansatz wurde von Dote et al. die Dichte-Verteilung einiger mit Kaonen gebundenen leichten Kerne berechnet und sehr hohe Dichten gefunden (siehe Fig. 16).

In $\mathrm{ppnK}^{-}$ist die mittlere Dichte das Dreifache einer normalen Kerndichte und falls es gelingt ppnK $\mathrm{K}^{-}$, also mit zwei Kaonen gebundene ${ }^{3}$ He Kerne zu erzeugen, übersteigt die mittlere Dichte die eines Kerns um das 6-fache. Dies sollte mit Schwerionen-Reaktionen oder Antiprotonen-Absorption im Rahmen des FAIR-Projekts der GSI durchaus möglich sein. Damit wäre die Möglichkeit eröffnet, kalte Kerne mit Dichten zu erzeugen, die man in Neutronensternen erwartet und die zudem den Bereich der Kaon-Kondensation erreichen.

Am KEK wurden $\mathrm{K}^{-}$Mesonen in einem ${ }^{4} \mathrm{He}$-Target gestoppt und die Energie der dabei emittierten Neutronen und Protonen gemessen. Dabei wurden erste Hinweise auf 


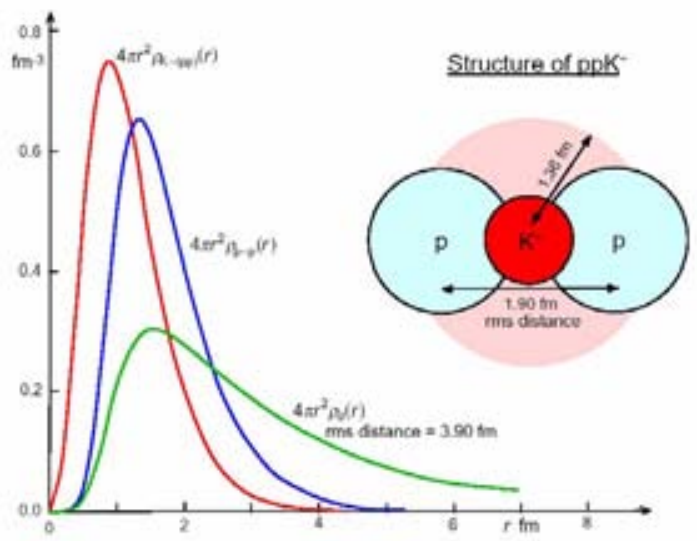

Fig. 15: Struktur von kaonischem Wasserstoff-Molekül.

mit Kaonen tiefgebundene Tribaryon-Zustände (ppnK ${ }^{-}$, pnnK ${ }^{-}$) mit Bindungsenergien von 173, bezw. $194 \mathrm{MeV}$ und Breiten kleiner als $20 \mathrm{MeV}$ gefunden. Damit sind die Bindungsenergien wesentlich höher als die $\Sigma \pi$-Zerfalls-Schwelle, was die geringe Zerfallsbreite beider Zustände erklärt. Die Bindungsenergien sind aber auch fast doppelt so hoch wie die theoretischen Voraussagen, was durch Berücksichtigung relativistischer Effekte und der Abnahme der effektiven Kaon Masse im dichten Kernmedium erklärt werden kann.

Die vier theoretisch erwarteten Tribaryon Zustände $(\mathrm{T}=0, \mathrm{~T}=1)$ sind mit ihren BindungsVerhältnissen in Fig. 17 dargestellt.

Besonders interessant wäre der noch ausstehende Nachweis des $\mathrm{pppK}^{-}$Systems mit 3 Protonen, wobei sich ein Proton in einem p-Zustand befände. Durch Studium seines Drei-Körper Zerfalls, könnte der Drehimpuls sowie die Größe des Kerns und damit seine Dichte bestimmt werden. In sehr vorläufigen Ergebnissen von d- $\Lambda$-Korrelationen wurden bei 1.9A GeV Ni-Ni Reaktionen mit dem FOPI Detektor der GSI in der Tat unabhängige Hinweise auf die Existenz und Bildung des ppnK ${ }^{-}$Systems in Schwerionen Reaktionen gefunden (Fig. 18).

Mit einem in Vorbereitung befindlichen Schwerionen Experiment, soll mittels $\mathrm{Al}-\mathrm{Al}$ Reaktionen bei 2.0A GeV, mit besserem Signal zu Untergrund Verhältnissen, das Ni-Ni Resultat bestätigt und nach anderen tief gebundenen $\mathrm{K}^{-}$Kernzuständen gesucht werden. Ferner soll mit dem FOPI Detektor in einer völlig exklusiven Messung, d.h. dem Nachweis aller Reaktions- und Zerfalls-Produkte im Ausgangskanal, das ppK ${ }^{-}$Molekül nachgewiesen werden. 


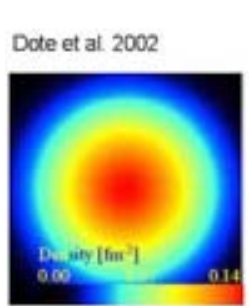

Single-K

Double-K
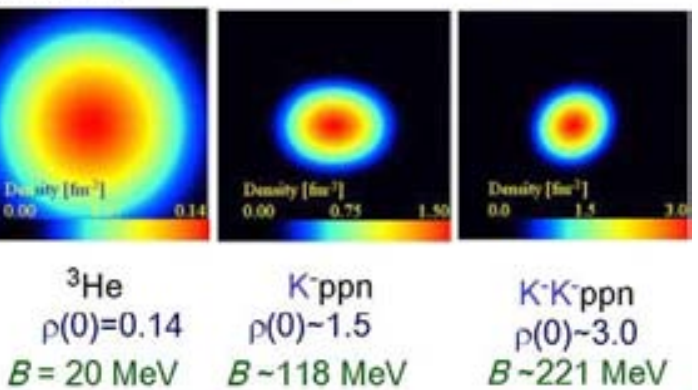

K·K'ppn

$\rho(0) \sim 3.0$

$B=20 \mathrm{MeV}$

$B \sim 118 \mathrm{MeV}$

$B \sim 221 \mathrm{MeV}$

Fig. 16: Vergleich der Dichteverteilung eines ${ }^{3}$ He Kerns mit durch 1 oder 2 Kaonen gebundenen ${ }^{3} \mathrm{He}$ Kernen.

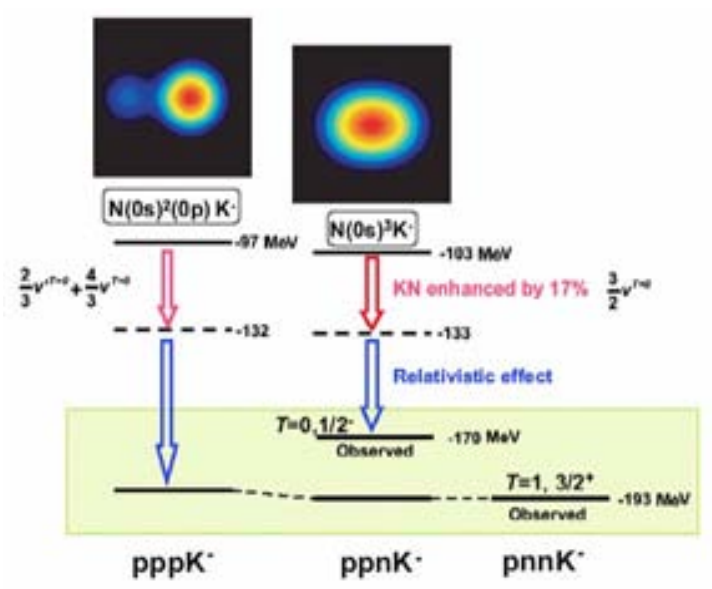

Fig. 17: Seltsame Tribaryonzustände (pppK $\left.{ }^{-}, \mathrm{ppnK}^{-}, \mathrm{pnnK}^{-}\right)$.

\section{Zusammenfassung und Ausblick}

In dem Vortrag wurden ausführlich die Eigenschaften von pionischen und kaonischen Atomen als Präzisions-Werkzeuge zur Untersuchung der Meson-Nukleon und Meson-Kern Wechselwirkung bei niedrigen Energien dargestellt. Durch Vergleich der Pion-Nukleon mit der Pion-Kern Wechselwirkung gelang es zum ersten Mal beim Studium der chiralen Dynamik die teilweise Restaurierung der spontan gebrochenen chiralen Symmetrie wechselwirkenden leichter Quarks, nachzuweisen. Wir stehen auch am Anfang eines hochinter- 

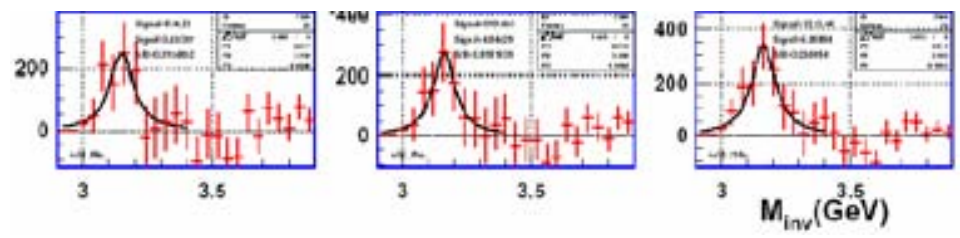

Fig. 18: Invariante Massen-Spektren von d- $\Lambda$ Zerfällen beobachtet in $1.9 \mathrm{GeV}$ Ni-Ni Reaktionen.

essanten Phänomens der starken Antikaon-Proton Wechselwirkung, die benutzt werden kann, im Labor hoch verdichtete Materie zu erzeugen, mit negativen Kaonen als Austauschteilchen.

\section{Epilog}

Wenn es Rosen sind werden sie blühen.

Herrn Zmeskal danken wir für die Überredung diesen Vortrag zu halten. Allen Mitgliedern des Stefan Meyer Instituts bin ich (PK) für die gute Zusammenarbeit während meiner Wiener Zeit zu großem Dank verpflichtet. Der Österreichischen Akademie der Wissenschaften danke ich (PK) für die stete Unterstützung bei meiner Aufgabe, allen voran Herrn Präsidenten Professor Mang und Herrn Präsidial-Direktor Dr. Vogel.

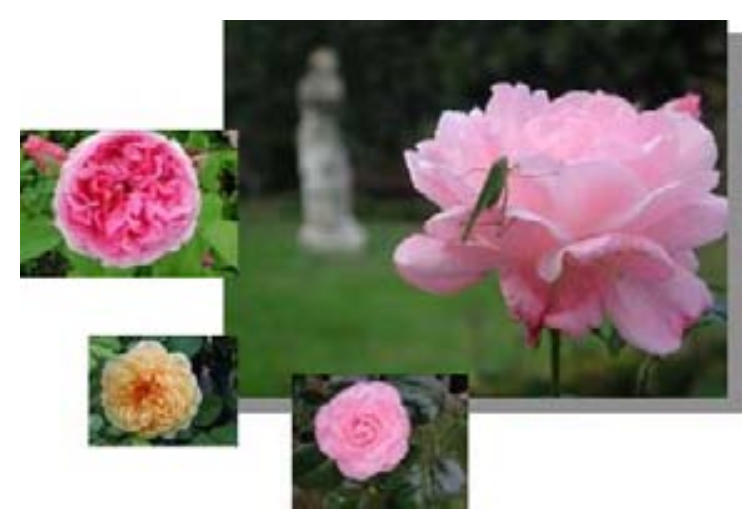


